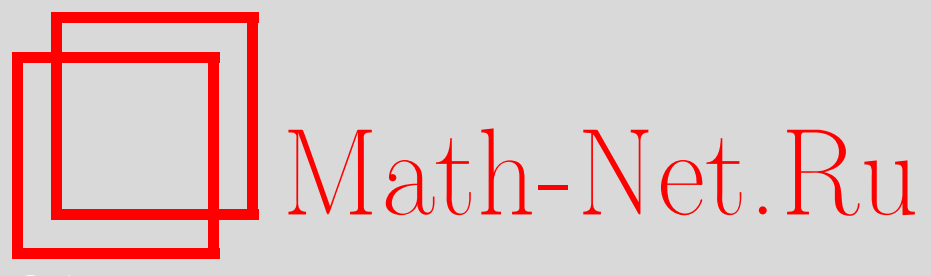

С. Де Лилло, Д. Лупо, М. Соммакал, Решения нелинейной задачи теплопроводности на полупрямой, $T M \Phi$, 2007, том 152, номер 1, 58-65

DOI: https://doi.org/10.4213/tmf6070

Использование Общероссийского математического портала Math-Net.Ru подразумевает, что вы прочитали и согласны с пользовательским соглашением http://www . mathnet.ru/rus/agreement

Параметры загрузки:

IP : 54.174 .149 .18

26 апреля 2023 г., 03:15:28

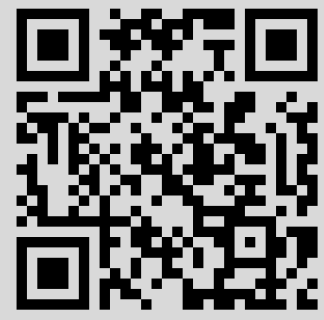




\title{
РЕШЕНИЯ НЕЛИНЕЙНОЙ ЗАДАЧИ ТЕПЛОПРОВОДНОСТИ НА ПОЛУПРЯМОЙ
}

\begin{abstract}
На полупрямой решается задача для нелинейного диффузионного уравнения с заданной зависящей от времени теплопроводностью в начале координат. Задача сводится к линейному интегральному уравнению Вольтерра, которое решается с помощью процесса Пикара последовательных приближений. Некоторые явные примеры проанализированы численно.
\end{abstract}

Ключевые слова: нелинейный, теплопроводность, полупрямая.

Нелинейные эволюционнные уравнения диффузионного типа в прошлом были предметом многочисленных исследований из-за их актуальности в приложениях (см. монографию [1] и библиографию в ней). В частности, нелинейное диффузионное уравнение

$$
u_{t}=\left(\frac{u_{x}}{u^{2}}\right)_{x}, \quad u \equiv u(x, t),
$$

широко изучалось в литературе как математическая модель, описывающая теплопроводность в высокополимерных системах [2] и в материалах типа материалов Сторма [3].

В работе [4] внимание было сосредоточено на задаче на полупрямой с начальным/граничным условием для уравнения (1) в случае заданной постоянной теплопроводности в начале координат.

Цель настоящей работы заключается в том, чтобы обобщить результаты работы [4], предложив решение приведенной выше задачи Коши для случая, когда теплопроводность на границе зависит от времени. Ниже мы покажем, что с помощью преобразования годографа нелинейную задачу для уравнения (1) можно свести к задаче типа задачи Робина для линейного уравнения теплопроводности с движущейся границей. Эта задача затем решается, т.е. редуцируется к линейному интегральному уравнению Вольтерра второго рода, которое допускает единственное решение. Мы также численно анализируем некоторые явные примеры.

*Dipartimento di Matematica e Informatica, Universitá degli Studi di Perugia, Via Vanvitelli 1, 06123 Perugia, Italy; Istituto Nazionale di Fisica Nucleare, Sezione di Perugia, Perugia, Italy. E-mail: Gaia.Lupo@pg.infn.it

${ }^{\dagger}$ Laboratoire J.-L. Lions, Université Pierre et Marie Curie, Paris VI, France. 
Начнем с рассмотрения задачи с начальным/граничным условием для уравнения (1) на полупрямой $0 \leqslant x<\infty$, характеризуемой начальным и граничными условиями

$$
\begin{gathered}
u(x, 0)=u_{0}(x), \quad 0 \leqslant x<\infty, \\
u(\infty, t)=\gamma, \quad u_{x}(\infty, t)=0, \quad t \geqslant 0, \\
\frac{u_{x}(0, t)}{u^{2}(0, t)}=f(t), \quad t \geqslant 0,
\end{gathered}
$$

где $\gamma$ - положительная константа, а $f(t)$ - интегрируемая функция. Граничное условие (2в) представляет заданную теплопроводность в начале координат (см. работу [4]).

Введем преобразование годографа

$$
u(x, t)=[v(z, t)]^{-1},
$$

где

$$
\begin{gathered}
\frac{\partial z}{\partial x}=u(x, t), \\
\frac{\partial z}{\partial t}=-\left(\frac{1}{u(x, t)}\right)_{x},
\end{gathered}
$$

условие совместности которого $\partial^{2} z / \partial x \partial t=\partial^{2} z / \partial t \partial x$ гарантировано уравнением (1).

Используя приведенное выше преобразование, отобразим уравнение (1) в линейное уравнение теплопроводности

$$
v_{t}=v_{z z}
$$

в области $F(t) \leqslant z<\infty$, где $F(t)$ удовлетворяет соотношению

$$
F(t)=\int_{0}^{t} f\left(t^{\prime}\right) d t^{\prime} .
$$

С помощью преобразования годографа мы свяжем с уравнением (4) начальные данные

$$
v(z, 0) \equiv v_{0}\left(z_{0}\right)=\left[u_{0}(x)\right]^{-1},
$$

где $z_{0}$ в силу уравнений (3б) и (3в) имеет вид

$$
z_{0} \equiv z_{0}(x)=\int_{0}^{x} u_{0}\left(x^{\prime}\right) d x^{\prime},
$$

а также граничные условия

$$
\begin{gathered}
v(\infty, t)=\frac{1}{\gamma}, \quad v_{z}(\infty, t)=0, \\
f(t) v(F(t), t)+v_{z}(F(t), t)=0 .
\end{gathered}
$$

Тогда задача с начальным/граничным условием для нелинейного диффузионного уравнения (1) с начальными данными (2а) и граничными условиями (2б), (2в) 
отображается в линейное уравнение теплопроводности (4) в области с движущейся границей, характеризующейся начальным условием (6a) и граничными условиями (7a), (7б).

Чтобы решить линейную задачу, введем фундаментальное ядро теплопроводности

$$
K\left(z-\xi, t-t^{\prime}\right)=\frac{1}{2 \sqrt{\pi}} \frac{1}{\sqrt{t-t^{\prime}}} e^{-(z-\xi)^{2} /\left(4\left(t-t^{\prime}\right)\right)}
$$

и проинтегрируем тождество Грина для уравнения теплопроводности

$$
\frac{\partial}{\partial \xi}\left(K \frac{\partial v}{\partial \xi}-v \frac{\partial K}{\partial \xi}\right)-\frac{\partial}{\partial t^{\prime}}(K v)=0
$$

по области $F\left(t^{\prime}\right)<\xi<\infty, \varepsilon<t^{\prime}<t-\varepsilon$, а также возьмем $\varepsilon \rightarrow 0$. Используя условие (7б) и тот факт, что $K(z-\xi, 0)=\delta(z-\xi)$, получаем

$$
v(z, t)=\int_{F(0)}^{+\infty} K(z-\xi, t) v_{0}(\xi) d \xi+\int_{0}^{t} K_{\xi}\left(z-F\left(t^{\prime}\right), t-t^{\prime}\right) v\left(F\left(t^{\prime}\right), t^{\prime}\right) d t^{\prime}
$$

Из уравнения (10) ясно, что можно определить $v(z, t)$, если известно граничное условие $v(F(t), t)$; поэтому удобно вычислить $(10)$ при $z=F(t)$. Полагая $w(t)=$ $v(F(t), t)$, получим

$$
w(t)=G(t)+\lambda \int_{0}^{t} R\left(t, t^{\prime}\right) w\left(t^{\prime}\right) d t^{\prime}
$$

где

$$
\begin{gathered}
G(t)=\int_{F(0)}^{+\infty} K(F(t)-\xi, t) v_{0}(\xi) d \xi \\
R\left(t, t^{\prime}\right)=\frac{1}{\lambda} K_{\xi}\left(F(t)-F\left(t^{\prime}\right), t-t^{\prime}\right), \quad \lambda=\frac{1}{4 \sqrt{\pi}} .
\end{gathered}
$$

Уравнение (11a) является линейным интегральным уравнением Вольтерра второго рода с сингулярным ядром $R\left(t, t^{\prime}\right)$. Подходящий выбор функции $f(t)$ позволяет с помощью уравнения (5) получить умеренно сингулярное ядро. Тогда линейное уравнение Вольтерра (11a) допускает единственное решение в предположении, что $G(t)$ является интегрируемой и ограниченной функцией своего аргумента [5]. Используя процесс Пикара последовательных приближений [5], решение уравнения (11a) можно записать как

$$
w(t)=G(t)+\lambda \int_{0}^{t} H\left(t, t^{\prime} ; \lambda\right) G\left(t^{\prime}\right) d t^{\prime}
$$

Здесь $H\left(t, t^{\prime} ; \lambda\right)$ - ядро резолъвенты, задаваемое рядом

$$
H\left(t, t^{\prime} ; \lambda\right)=\sum_{n=0}^{\infty} \lambda^{n} R_{n+1}\left(t, t^{\prime}\right)
$$




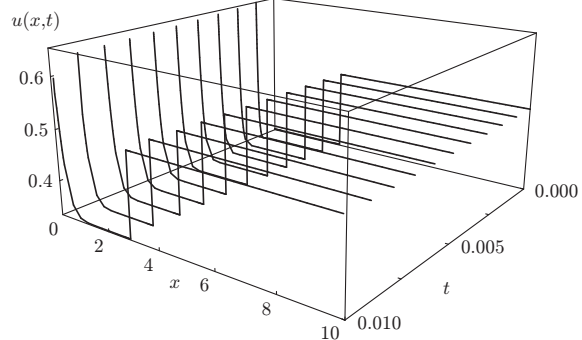

$a$

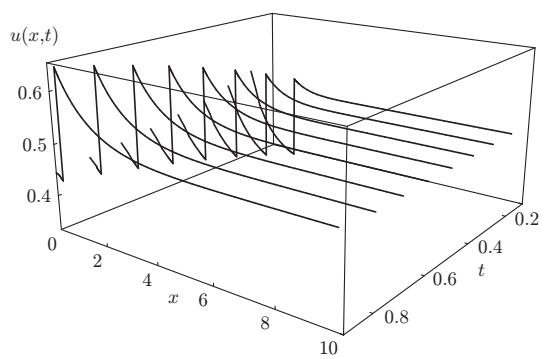

8

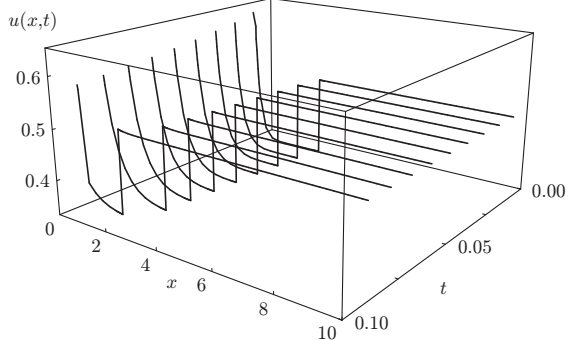

б

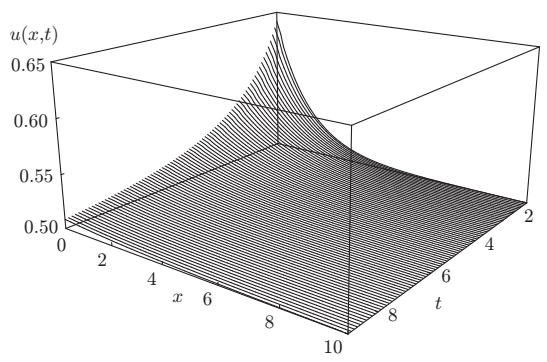

Рис. 1. Графическое представление решения $u(x, t)$, соответствующего примеру 1 (см. (18)), построенное относительно переменной $x$ при фиксированных значениях $t$ для различных интервалов: $0 \leqslant t \leqslant 0.01$ (а), $0.01 \leqslant t \leqslant 0.09$ (б), $0.2 \leqslant t \leqslant 0.9$ (в), $1.0 \leqslant t \leqslant 10$ (г).

где

$$
\begin{gathered}
R_{n+1}\left(t, t^{\prime}\right)=\int_{0}^{t} R\left(t, t^{\prime \prime}\right) R_{n}\left(t^{\prime \prime}, t^{\prime}\right) d t^{\prime \prime}, \quad n=1,2,3, \ldots \\
R_{1}\left(t, t^{\prime}\right)=R\left(t, t^{\prime}\right)
\end{gathered}
$$

Ниже мы численно исследуем четыре примера, соответствующие двум различным выборам функции $f(t)$ (см. условие $(2$ в)): в первом случае $f(t)$ является константой, а во втором - линейной функцией времени:

$$
\begin{gathered}
f(t)=\alpha, \quad \alpha \in \mathbb{R} ; \\
f(t)=a t+b, \quad a, b \in \mathbb{R} .
\end{gathered}
$$

Из (14а) и (14б) ясно, что с учетом соотношения (5) $F(t)$ является соответственно линейной или квадратичной функцией времени. Заметим также, что выбор (14a) соответствует случаю, который аналитически рассмаривался в работе [4]. В обоих случаях мы рассматриваем начальные даннные $u_{0}(x)$, совместные с асимптотическим условием (2б), соответствующим, во-первых, функции

$$
u_{0}(x)=\beta-(\beta-\gamma) \vartheta(x-\eta), \quad \beta, \eta \in \mathbb{R},
$$



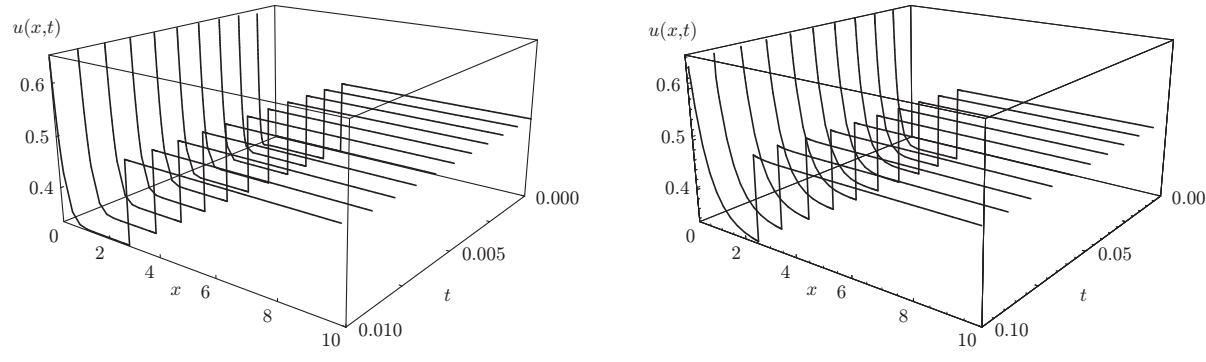

$a$

$\sigma$
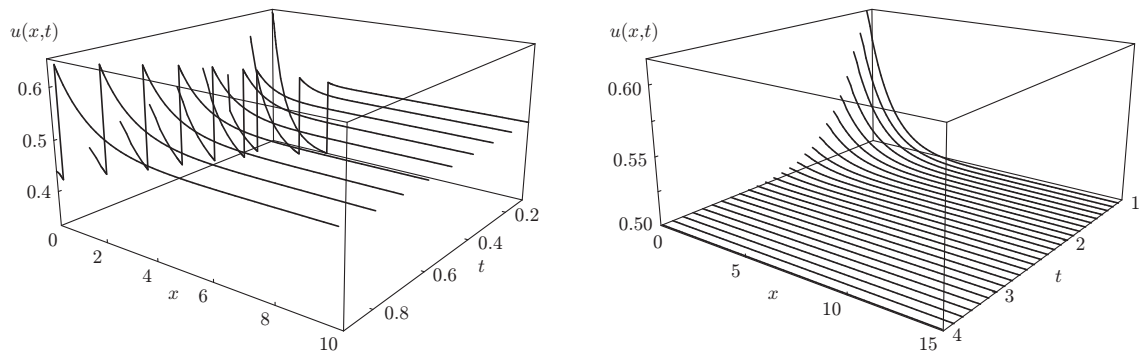

B

2

Рис. 2. Графическое представление решения $u(x, t)$, соответствующего примеру 2 (см. (19)), построенное относительно переменной $x$ при фиксированных значениях $t$ для различных интервалов: $0 \leqslant t \leqslant 0.01$ (а), $0.01 \leqslant t \leqslant 0.09$ (б), $0.1 \leqslant t \leqslant 0.9$ (в), $1.1 \leqslant t \leqslant 3.6$ (г).

где $\vartheta(x)$ - обычная единичная ступенчатая функция, а во-вторых, функции

$$
u_{0}(x)=\frac{\gamma}{1+W\left(c \gamma e^{-\gamma(\kappa x-c)}\right)}, \quad \kappa, c \in \mathbb{R},
$$

где $W(x)-W$-функция Ламбера, неявно определяемая соотношением $f(W)=W e^{W}$.

В первом случае с $F(t)$, определяющейся (14a), наш метод состоит в прямом вычислении функции $v(z, t)$ через явное решение, как это было показано в работе [4]. Затем мы вычисляем функцию $v(z, t)$ в соответствии с выражением (10) и окончательно получаем решение $u(x, t)$, обращая преобразование годографа (3а)-(3в). При фиксированном времени $t=t^{*}$ с помощью (3а) и (3б) получаем

$$
\left.x(z)\right|_{t^{*}}=\int_{0}^{z} v\left(z^{\prime}, t^{*}\right) d z^{\prime}
$$

Тогда из выражения (16) мы получаем обратную функцию $\left.z(x)\right|_{t^{*}}$ и окончательно находим решение исходной задачи:

$$
u\left(x, t^{*}\right)=\left[v\left(\left.z(x)\right|_{t^{*}}, t^{*}\right)\right]^{-1},
$$

в соответствии с (3a). 

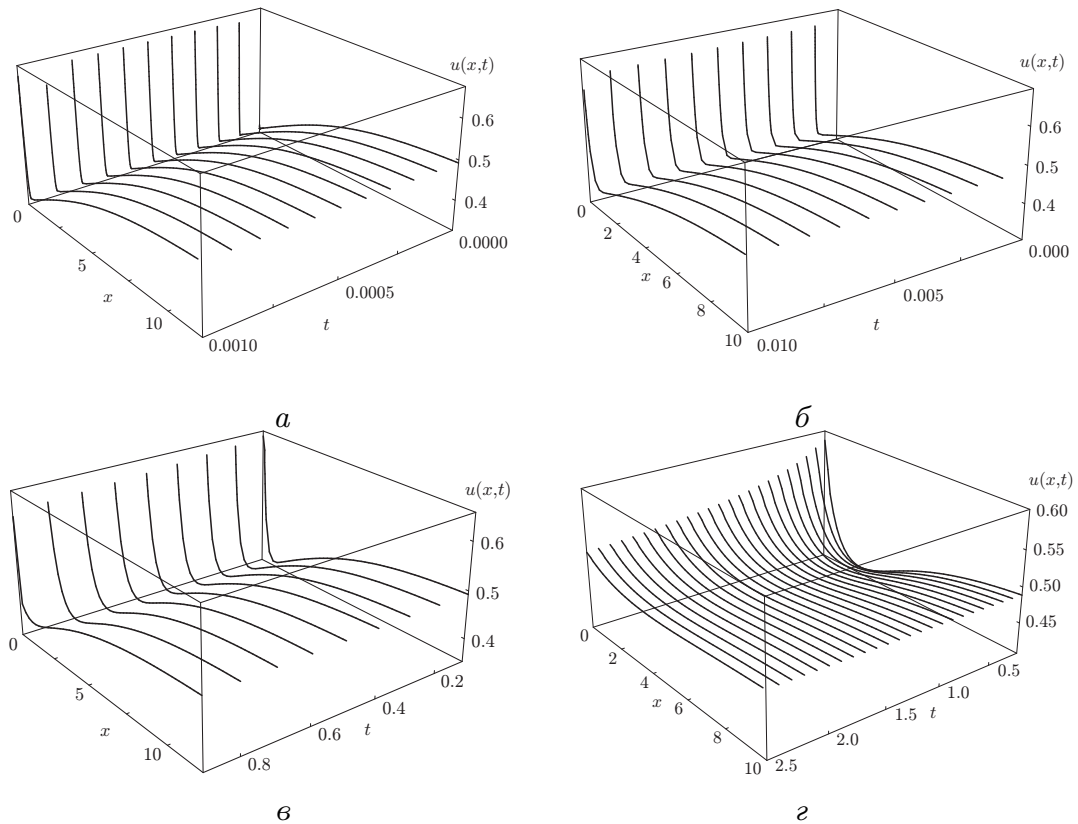

Рис. 3. Графическое представление решения $u(x, t)$, соответствующего примеру 3 (см. (20)), построенное относительно переменной $x$ при фиксированных значениях $t$ для различных интервалов: $0 \leqslant t \leqslant 0.001$ (а), $0.001 \leqslant t \leqslant 0.01$ (б), $0.01 \leqslant t \leqslant 0.09$ (в), $0.2 \leqslant t \leqslant 2.5$ (г).

Если $F(t)$ определяется (14б), то интегральное уравнение Вольтерра (11a) не решается в квадратурах, как в предыдущем случае, однако оно должно решаться численно. Решение $v(z, t)$ линейной задачи получается с помощью уравнения (10), но, конечно, вычислительные издержки такого алгоритма гораздо больше, чем в предыдущем случае. Интегральное уравнение (10) интегрируется численно при использовании неравномерного fixed-mesh-метода, с тем чтобы избежать проблем, связанных с наличием умеренно сингулярного ядра (см., например, книгу [6] и ссылки в ней). Как объяснялось выше, после вычисления функции $v(z, t)$ мы, обращая преобразование годографа, получаем решение $u(x, t)$ нелинейной задачи (см. (16) и (17)).

Ниже мы подробно анализируем примеры и интерпретируем численные результаты, представив ряд графиков. Подчеркнем, что на всех графиках каждая линия представляет собой функцию $u(x, t)$ в фиксированный момент времени. Как и ожидалось, при больших $x$ решение нелинейной задачи $u(x, t)$ асимптотически приближается к значению $\gamma$.

ПримеР 1. Функция $u_{0}(x)$ задается уравнением (15a), а $f(t)$ - уравнением (14a), где $\alpha=1, \beta=1 / 3, \eta=3$ и $\gamma=1 / 2$. Тогда

$$
u_{0}(x)=\frac{1}{6}[2+\vartheta(x-3)], \quad f(t)=1 .
$$




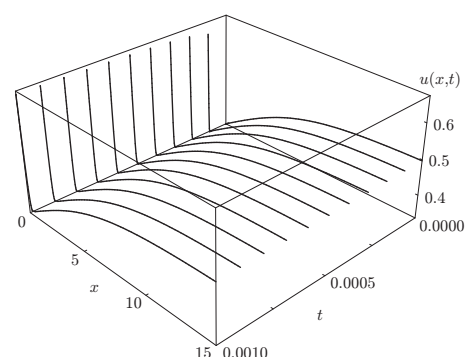

$a$

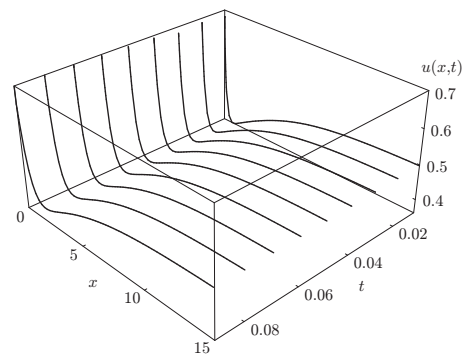

8

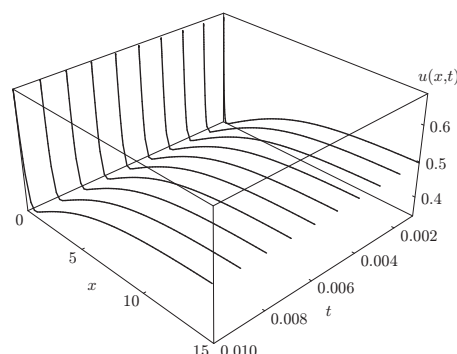

$\sigma$

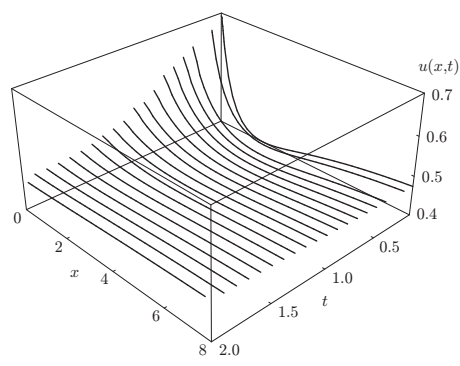

2

Рис. 4. Графическое представление решения $u(x, t)$, соответствующего примеру 4 (см. (22)), построенное относительно переменной $x$ при фиксированных значениях $t$ для различных интервалов: $0 \leqslant t \leqslant 0.001$ (а), $0.001 \leqslant t \leqslant 0.01$ (б), $0.01 \leqslant t \leqslant 0.09$ (в), $0.1 \leqslant t \leqslant 2$ (г).

Результаты численного моделирования представлены на рис. 1 . Видно, что при $0<$ $t<1$ разрыв решения $u(x, t)$ по переменной $x$, обусловленный выбором ступенчатой функции $\vartheta(x)$ в начальных данных $u_{0}(x)$, сдвигается к началу координат вдоль оси $x$ с ростом $t$.

ПримеР 2. Функция $u_{0}(x)$ задается уравнением (15a), а $f(t)$-уравнением (14б), где $a=2, b=0, \beta=1 / 3, \eta=3$ и $\gamma=1 / 2$. Тогда

$$
u_{0}(x)=\frac{1}{6}[2+\vartheta(x-3)], \quad f(t)=2 t .
$$

Результаты численного моделирования представлены на рис. 2. Сравнивая этот результат с предыдущим, мы видим, что выбор функции (14б) (а именно квадратичной по времени функции $F(t))$, по-видимому, приводит к более быстрому по времени приближению решения $u(x, t)$ к постоянной функции $\hat{u}(x, t)=\gamma$.

ПримеР 3. Функция $u_{0}(x)$ задается уравнением (15б), а $f(t)$ - уравнением (14a), где $\alpha=1, c=1, \kappa=2 / 3$ и $\gamma=1 / 2$. Тогда

$$
u_{0}(x)=\frac{1}{2}\left[1+W\left(\frac{1}{2} e^{-x / 3+1 / 2}\right)\right]^{-1}, \quad f(t)=1 .
$$


В этом случае полезно заметить, что из уравнения (20) с помощью уравнений (6а) и (6б) мы получаем

$$
v_{0}(z)=\frac{1}{\gamma}+c e^{-\kappa z}=2+e^{-2 z / 3} .
$$

Результаты численного моделирования представлены на рис. 3.

ПримеР 4. Функция $u_{0}(x)$ задается формулой (15б), а $f(t)$ - формулой (14б), где $\alpha=1, c=1, \kappa=2 / 3$ и $\gamma=1 / 2$. Тогда

$$
u_{0}(x)=\frac{1}{2}\left[1+W\left(\frac{1}{2} e^{-x / 3+1 / 2}\right)\right]^{-1}, \quad f(t)=2 t .
$$

Результаты численного моделирования представлены на рис. 4. Сравнивая этот результат с предыдущим, мы видим, что выбор функции (14б) (а именно квадратичной по времени функции $F(t))$, по-видимому, приводит к более быстрому по времени приближению решения $u(x, t)$ к постоянной функции $\hat{u}(x, t)=\gamma$.

\section{Список литературы}

[1] P. L. Sachdev, Nonlinear Diffusive Waves, Cambridge Univ. Press, Cambridge, 1987.

[2] G. Bluman, S. Kumei, J. Math. Phys., 21 (1980), 1019.

[3] M. L. Storm, J. Appl. Phys., 22 (1951), 940.

[4] S. De Lillo, G. Lupo, G. Sanchini, J. Phys. A, 39 (2006), 7299.

[5] Ф. Трикоми, Дифференциальные уравнения, ИЛ, М., 1960.

[6] W. H. Press, S. A. Teukolsky, W. T. Vetterling, B. P. Flannery, Numerical Recipes in Fortran. The Art of Scientific Computing, Cambridge Univ. Press, Cambridge, 1992.

3 Теоретическая и математическая физика, т. 152, № 1, 2007 г. 